\title{
ROBUST MIMO COGNITIVE RADIO SYSTEMS UNDER TEMPERATURE INTERFERENCE CONSTRAINTS
}

\author{
Yang Yang ${ }^{1}$, Peiran Song ${ }^{2}$, Gesualdo Scutari ${ }^{2}$, Daniel P. Palomar ${ }^{1}$
}

1. Dept. of Electronic and Computer Eng., Hong Kong Univ. of Sci. \& Tech., Hong Kong. Emails: \{yangyang,palomar\}@ust.hk

2. Dept. of Electrical Eng., State Univ. of New York (SUNY), Buffalo, NY 14260, USA. Emails:\{peiranso,gesualdo\}@buffalo.edu

\begin{abstract}
In cognitive radio (CR) systems, the primary users (PU) are protected by temperature interference constraints imposed on secondary users (SU). However, such limitations may be easily violated by SUs if perfect SU-to-PU channel state information (CSI) is not available at the secondary transmitters. In this paper, we propose a novel and distributed design of MIMO CR networks that is robust against imperfect SU-to-PU CSI. Specifically, we formulate the system design as a noncooperative game and robust global interference constraints are enforced via pricing; the prices are thus additional variables to be optimized. Building on the advanced and new theory of finitedimensional variational inequalities (VI) in the complex domain, we analyze the proposed NE problem and devise alternative distributed algorithms along with their convergence properties.
\end{abstract}

Index Terms - Cognitive Radio System, MIMO System, Temperature Interference Constraints, Variational Inequalities, WorstCase Robust Design.

\section{INTRODUCTION}

Cognitive Radio (CR) $[1,2,3]$ is a promising technique that allows flexible and efficient usage of the scarce radio spectrum, which is, however, underutilized by the current fixed spectrum assignmen$\mathrm{t}$ policies. The design of secondary user $\mathrm{CR}$ systems has been addressed in a number of works in different scenarios such as $[4,5,6$, $7,8,9,10]$, based on either centralized network utility maximization philosophy $[7,8,10]$ or decentralized game theoretical formulations $[4,5,6]$. The quality of service (QoS) of the PUs is guaranteed by imposing interference constraints to the SUs; either local interference constraints (i.e. at the level of each SU) $[4,9]$ or global interference constraints (i.e., on the overall interference generated by all the SUs) $[5,6,7,8,10]$ have been adopted. In the context of MIMO systems, the analysis is mainly limited to local interference constraints $[4,9]$ with the exception of [8], whose proposed algorithms, however, cannot be implemented in a distributed way. Moreover, all the aforementioned papers (except [9] in the case of local interference constraints and [10] in the case of MISO multicast network with only one secondary transmitter) assume perfect channel state information between SUs and PUs (SU-to-PU CSI), which is not realistic in a real CR scenario, due, e.g., to inaccurate or limited CSI at the secondary transmitters.

In this paper, we consider for the first time a distributed design of MIMO cognitive radio systems (composed of an arbitrary number of PUs and SUs) under worst-case robust global interference constraints. Aiming at finding distributed algorithms, we formulate the system design as a noncooperative game, where each SU

The work of Yang and Palomar is supported by the Hong Kong RGC 617911 research grant. The work of Song and Scutari is supported by the NSF grant No. CNS-1218717. competes against the others to maximize his own transmission rate subject to the robust global interference constraints as well as power constraints. In order to keep the design as decentralized as possible, the robust global interference constraints are imposed via a proper pricing mechanism; the prices are thus additional variables to be optimized. At equilibrium, they are required to be complementary to the respective robust interference constraints; that is, a price is positive only if the robust constraint is violated.

The presence of possibly unbounded price variables and pricing clearing conditions associated with the robust global interference constraints as well as the MIMO setting (each user's strategies are complex matrices) make the analysis of the resulting game a challenging task. To deal with these difficulties, we hinge on the recently developed theory of complex variational inequalities [11]. Building on this theory, we study the existence and uniqueness of the $\mathrm{NE}$ as well as design distributed algorithms along with their convergence properties. We also investigate the impact of error in pricing update on the convergence of proposed distributed algorithms. Numerical results show that our novel game-theoretical formulation based on robust global interference constraints outperforms (in terms of achievable sum-rate) current decentralized state-of-the-art design$\mathrm{s}$ based on (robust) local interference constraints [9]. This new line of analysis based on VIs in the complex domain is also expected to be broadly applicable to other game theoretical formulations with complex optimization variables.

\section{SYSTEM MODEL AND PROBLEM FORMULATION}

Consider a hierarchical MIMO CR system composed of $K$ PUs sharing the licensed spectrum with a network of $Q$ SUs, modeled as a MIMO Gaussian Interference Channel (IC). Each SU $q$ is equipped with $N_{q}$ and $M_{q}$ transmit and receive antennas, respectively, and PU $k$ has $M_{k}$ receive antennas. Let $\mathbf{H}_{r q} \in \mathbb{C}^{M_{r} \times N_{q}}$ (resp. $\mathbf{G}_{k q} \in$ $\mathbb{C}^{M_{k} \times N_{q}}$ ) be the cross-channel between SU $q$ and SU $r$ (resp. PU $k$ ). Under basic information theoretical assumptions, the transmission rate of SU $q$ can be written as

$$
R_{q}\left(\mathbf{Q}_{q}, \mathbf{Q}_{-q}\right) \triangleq \log \operatorname{det}\left(\mathbf{I}+\mathbf{H}_{q q}^{H} \mathbf{R}_{-q}\left(\mathbf{Q}_{-q}\right)^{-1} \mathbf{H}_{q q} \mathbf{Q}_{q}\right)
$$

where $\mathbf{Q}_{q}$ is the transmit covariance matrix of $\mathrm{SU} q, \mathbf{Q}_{-q} \triangleq$ $\left(\mathbf{Q}_{r}\right)_{r \neq q}, \mathbf{R}_{-q}\left(\mathbf{Q}_{-q}\right) \triangleq \mathbf{R}_{n_{q}}+\sum_{r \neq q} \mathbf{H}_{q r} \mathbf{Q}_{r} \mathbf{H}_{q r}^{H}$ with $\mathbf{R}_{n_{q}} \succ \mathbf{0}$ being the covariance matrix of the noise plus the interference from the PUs. The feasible set of SU $q$ is

$$
\mathcal{Q}_{q} \triangleq\left\{\mathbf{Q}_{q} \succeq \mathbf{0}: \operatorname{tr}\left(\mathbf{Q}_{q}\right) \leq P_{q}\right\}
$$

where $P_{q}$ is the total transmit power in units of energy per transmission. Other (convex) power constraints such as peak and per-antenna average power constraint can be readily incorporated without affecting the forthcoming analysis and results.

In addition to power budget constraints, the SUs are subject to global interference constraints that are imposed to protect the QoS of 
PUs. We model inaccurate or limited SU-to-PU CSI following the widely used approach in $[9,12,13,14,15]$ : the real channel $\mathbf{G}_{k q}$ lies in the neighborhood of a nominal channel $\widehat{\mathbf{G}}_{k q}{ }^{1}$. This leads to the definition of the so-called uncertainty region:

$$
\mathcal{U}_{k q} \triangleq\left\{\mathbf{G}_{k q} \in \mathbb{C}^{M_{k} \times N_{q}}:\left\|\mathbf{G}_{k q}-\widehat{\mathbf{G}}_{k q}\right\|_{\mathbf{T}_{k q}, F} \leq \varepsilon_{k q}\right\}
$$

with $\varepsilon_{k q}$ is the (given) radius of the region, and $\mathbf{T}_{k q}$ is a given positive definite matrix. It follows from (3) that the worst-case global aggregate interference constraint imposed to the SUs by each PU $k$ is

$$
\sum_{q=1}^{Q} \phi_{k q}\left(\mathbf{Q}_{q}\right) \triangleq \sum_{q=1}^{Q} \max _{\mathbf{G}_{k q} \in \mathcal{U}_{k q}}\left\{\mathbf{G}_{k q} \mathbf{Q}_{q} \mathbf{G}_{k q}^{H}\right\} \leq I_{k},
$$

where $I_{k}$ is the maximum level of interference tolerable by PU $k$. Note that $\phi_{k q}\left(\mathbf{Q}_{q}\right)$ is convex but non-differentiable. We remark that other global interference constraints, including (directional) null constraints, interference constraints over specific directions $\mathbf{w}_{k}$ such as $\sum_{q=1}^{Q} \mathbf{w}_{k}^{H} \mathbf{G}_{k q} \mathbf{Q}_{q} \mathbf{G}_{k q}^{H} \mathbf{w}_{k}$, and peak average interference constraints such as $\sum_{q=1}^{Q} \lambda_{\max }\left(\mathbf{G}_{k q} \mathbf{Q}_{q} \mathbf{G}_{k q}^{H}\right)$, can be incorporated in the system and treated using the same methodology we are going to introduce, see [16] for more details.

System Design. Aiming at finding distributed solution algorithms, we formulate the secondary system design as a robust noncooperative game, where each $\mathrm{SU}$ aims at maximizing his transmission rate (1) subject to the power and robust interference constraints (2) and (4). To keep the system design as decentralized as possible, the robust global interference constraints are enforced by introducing a pricing term in the objective function of each user: there is one price $\mu_{k} \geq 0$ associated with each of the robust global interference constraints; the vector of all prices is denoted by $\boldsymbol{\mu} \triangleq\left(\mu_{k}\right)_{k=1}^{K}$. Stated in mathematical terms, we have the following priced Nash equilibrium problem (NEP): anticipating the rival strategies $\mathbf{Q}_{-q}$ and the price $\boldsymbol{\mu}$, each user $q$ solves

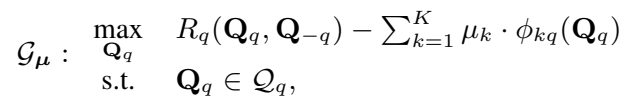

The game is completed with the side constraints to be satisfied by the price vector $\boldsymbol{\mu}$ :

$$
0 \leq \mu_{k} \perp I_{k}-\sum_{q=1}^{Q} \phi_{k q}\left(\mathbf{Q}_{q}\right) \geq 0, \quad \forall k=1, \ldots, K,
$$

where $a \perp b$ means $a \cdot b=0$. The complementary condition (6) says that users are penalized only when the resources become scarce; the price $\mu_{k}$ is 0 when the interference constraint is strictly satisfied.

Differently from $[5,9,17]$, our formulation incorporates robust global temperature interference constraint, and this leads to several new and nontrivial difficulties, namely: 1) each user's objective function is non-differentiable; 2) the pricing term is nonlinear in the optimization variables; 3 ) the optimization variables are complex matrices. Because of these issues, the NEP $\mathcal{G}_{\mu}$ together with (6) cannot be studied using existing methodologies $[5,9,17]$. Note also that even though each user's optimization problem is convex, the game may not have a NE because of the unboundedness of the price vector. We address these technical difficulties in following sections.

\footnotetext{
${ }^{1}$ An estimate of the nominal channel can be obtained at the secondary transmitters using standard signal processing techniques. For example, in a time-division duplex (TDD) mode under reciprocity assumption between the forward and backward channels, the nominal channel can be estimated by using the pilots sent by the PU receivers when they transmit [10].
}

\section{COMPLEX VARIATIONAL INEQUALITIES}

The first step of our analysis is to get rid of the non-differentiability of the users' optimization problems by rewriting (5) in the following equivalent form:

$$
\begin{array}{cl}
\widetilde{\mathcal{G}}_{\boldsymbol{\mu}}: & \max _{\mathbf{Q}_{q}, \mathbf{t}_{q}} \\
\text { s.t. } & R_{q}\left(\mathbf{Q}_{q}, \mathbf{Q}_{-q}\right)-\sum_{k=1}^{k} \mu_{k} t_{k q} \\
& \left(\mathbf{Q}_{q}, \mathbf{t}_{q}\right) \in \widetilde{\mathcal{Q}}_{q},
\end{array}
$$

where $\mathbf{t}_{q} \triangleq\left(t_{k q}\right)_{k=1}^{K}$ is a vector of slack variables and $\widetilde{\mathcal{Q}}_{q} \triangleq$ $\left\{\left(\mathbf{Q}_{q}, \mathbf{t}_{q}\right): \mathbf{Q}_{q} \in \mathcal{Q}_{q}, \phi_{k q}\left(\mathbf{Q}_{q}\right) \leq t_{k q}, \forall k=1, \ldots, K\right\}$. The complementary condition (6) can be rewritten as:

$$
0 \leq \mu_{k} \perp I_{k}-\sum_{q=1}^{Q} t_{k q} \geq 0, \quad \forall k=1, \ldots, K .
$$

We will denote the NEP $\widetilde{\mathcal{G}} \boldsymbol{\mu}$ with complementary condition (8) as $\widetilde{\mathcal{G}}$.

To deal with complex matrix variables, we hinge on the so-called complex VIs, recently introduced in [11], and rewrite the game $\widetilde{\mathcal{G}}$ as a complex VI. An alternative approach would be to write the users' optimization problems in terms of real and imaginary parts of the original complex variables, which however is very cumbersome and destroys the structure of the optimization problem.

Definition 1 Given a closed set $\mathcal{X} \in \mathbb{C}^{n \times m}$ and a complex-valued matrix function $\mathbf{F}^{\mathbb{C}}(\mathbf{X}): \mathcal{X} \ni \mathbf{X} \rightarrow \mathbb{C}^{n \times m}$, the complex VI problem, denoted by $\operatorname{VI}\left(\mathcal{X}, \mathbf{F}^{\mathbb{C}}\right)$, consists in finding a point $\mathbf{X}^{\star}$ such that ${ }^{2}$

$$
\left(\mathbf{X}-\mathbf{X}^{\star}\right) \bullet \mathbf{F}^{\mathbb{C}}\left(\mathbf{X}^{\star}\right) \geq 0, \quad \forall \mathbf{X} \in \mathcal{X},
$$

where $\mathbf{A} \bullet \mathbf{B} \triangleq \Re\left\{\operatorname{tr}\left(\mathbf{A}^{H} \mathbf{B}\right)\right\}$ and $\Re\{\bullet\}$ is the real part operator.

Define a complex-valued matrix function $\mathbf{F}^{\mathbb{C}}=\left(\mathbf{F}_{q}^{\mathbb{C}}\right)_{q=1}^{Q}$ and the joint strategy set $\mathcal{Q}$ as

$$
\begin{aligned}
& \mathbf{F}_{q}^{\mathbb{C}}(\mathbf{Q})=-\nabla_{\mathbf{Q}_{q}^{*}} R_{q}\left(\mathbf{Q}_{q}, \mathbf{Q}_{-q}\right), \\
& \mathcal{Q} \triangleq\left\{\left(\mathbf{Q}_{q}\right)_{q=1}^{Q}: \begin{array}{l}
\mathbf{Q}_{q} \in \mathcal{Q}_{q}, \quad \forall q=1, \ldots, Q, \\
\sum_{q=1}^{Q} \phi_{k q}\left(\mathbf{Q}_{q}\right) \leq I_{k}, \quad \forall k=1, \ldots, K,
\end{array}\right\},
\end{aligned}
$$

where the gradient is with respect to the complex conjugate of $\mathbf{Q}_{q}$.
We have the following connection between the game $\widetilde{\mathcal{G}}$ and the $\operatorname{VI}\left(\mathcal{Q}, \mathbf{F}^{\mathbb{C}}\right)[16]$.

Proposition 2 The game $\widetilde{\mathcal{G}}$ in (7)-(8) is equivalent to the $\operatorname{VI}\left(\mathcal{Q}, \mathbf{F}^{\mathbb{C}}\right)$, which always admits a solution. The equivalence is in the following sense: if $\mathbf{Q}^{\star} \triangleq\left(\mathbf{Q}_{q}^{\star}\right)_{q=1}^{Q}$ is a solution of the $\operatorname{VI}\left(\mathcal{Q}, \mathbf{F}^{\mathbb{C}}\right)$, then there exists $a \mathbf{t}^{\star} \triangleq\left(t_{k q}^{\star}\right)_{k, q}$ with $t_{k q}^{\star}=\phi_{k q}\left(\mathbf{Q}_{q}^{\star}\right)$ and $a \boldsymbol{\mu}^{\star} \triangleq\left(\mu_{k}^{\star}\right)_{k}$-the multiplier of the VI associated with (4)-such that $\left(\mathbf{Q}^{\star}, \mathbf{t}^{\star}, \boldsymbol{\mu}^{\star}\right)$ is an equilibrium pair of $\widetilde{\mathcal{G}}$. Conversely, if $\left(\mathbf{Q}^{\star}, \mathbf{t}^{\star}, \boldsymbol{\mu}^{\star}\right)$ is an equilibrium pair of $\widetilde{\mathcal{G}}$, then $t_{k q}^{\star}=\phi_{k q}\left(\mathbf{Q}_{q}^{\star}\right)$ and $\mathbf{Q}^{\star}$ is a solution of $\operatorname{VI}\left(\mathcal{Q}, \mathbf{F}^{\mathbb{C}}\right)$ with $\boldsymbol{\mu}^{\star}$ being the multiplier associated with (4).

The reformulation of the game $\widetilde{\mathcal{G}}$ as a VI plays a key role in studying the existence of the solution. However, it leads to algorithms that, in principle, require some coordination among the SUs, since the global interference constraints impose a coupling among strategies of the SUs (indeed the set $\widetilde{\mathcal{Q}}$ does not have a Cartesian structure).

${ }^{2}$ If $\mathbf{X}$ and $\mathbf{F}^{\mathbb{C}}(\mathbf{X})$ are partitioned according to $\mathbf{X}=\left(\mathbf{X}_{q}\right)_{q=1}^{Q}$ and $\mathbf{F}^{\mathbb{C}}(\mathbf{X})=\left(\mathbf{F}_{q}^{\mathbb{C}}(\mathbf{X})\right)_{q=1}^{Q}$ [such that $\mathbf{X}_{q} \bullet \mathbf{F}_{q}^{\mathbb{C}}(\mathbf{X})$ is well-defined for all $q=1, \ldots, Q]$, then (9) is interpreted as $\sum_{q=1}^{Q}\left(\mathbf{X}_{q}-\mathbf{X}_{q}^{\star}\right) \bullet \mathbf{F}_{q}^{\mathbb{C}}(\mathbf{X}) \geq$ $0, \forall \mathbf{X} \in \mathcal{X}$. 


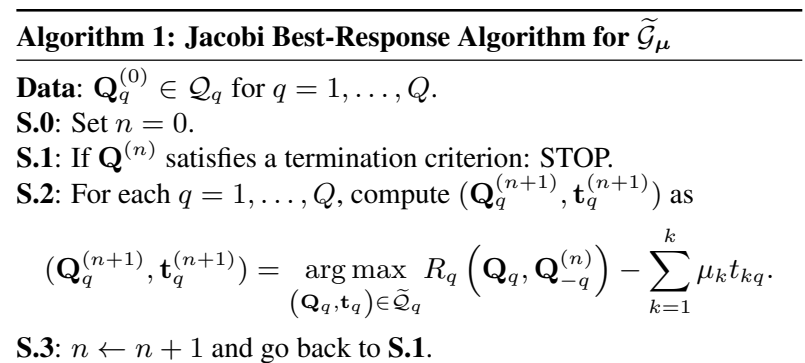

To deal with this issue, we rewrite the $\operatorname{VI}\left(\mathcal{Q}, \mathbf{F}^{\mathbb{C}}\right)$-the game $\widetilde{\mathcal{G}}$-in an equivalent but more convenient form. More specifically, denoting by $\left(\mathbf{Q}^{\star}(\boldsymbol{\mu}), \mathbf{t}^{\star}(\boldsymbol{\mu})\right)$ the NE of the game $\widetilde{\mathcal{G}}_{\boldsymbol{\mu}}$ for a given $\boldsymbol{\mu} \geq \mathbf{0}$, and introducing the worst-case interference violation function $\mathbf{M}(\boldsymbol{\mu})$ :

$$
\mathbf{M}(\boldsymbol{\mu}) \triangleq\left(I_{k}-\sum_{q=1}^{Q} t_{k q}^{\star}(\boldsymbol{\mu})\right)_{k=1}^{K},
$$

the game $\widetilde{\mathcal{G}}$ can be written in the following equivalent form [16]

Proposition 3 Suppose $\widetilde{\mathcal{G}}_{\boldsymbol{\mu}}$ has a unique NE for any given $\boldsymbol{\mu} \geq$ 0. Then $\widetilde{\mathcal{G}}$ is equivalent to the following nonlinear complementary problem $(N C P)$ :

$$
\mathbf{0} \leq \boldsymbol{\mu} \perp \mathbf{M}(\boldsymbol{\mu}) \geq \mathbf{0} .
$$

The NCP reformulation offers the possibility of devising iterative algorithms that can be implemented in a distributed fashion among all users and whose convergence can be studied using results from the theory of VIs. In the following, we preliminarily introduce distributed algorithms solving the game $\widetilde{\mathcal{G}}_{\mu}$ [which allows the computation of $\mathbf{M}(\boldsymbol{\mu})$ in (11)]; then, building on this result, we focus on distributed algorithms for the NCP (11) (and thus the original game $\widetilde{\mathcal{G}}$ ).

\section{DISTRIBUTED ALGORITHMS FOR $\widetilde{\mathcal{G}}_{\mu}$}

In this section we study distributed algorithms for the game $\widetilde{\mathcal{G}}_{\mu}$, given $\boldsymbol{\mu} \geq \mathbf{0}$. Before stating the main results, we introduce some preliminary definitions. A matrix $\mathbf{A}$ is called a $P$-matrix if every principle minor of $\mathbf{A}$ is positive (a positive definite matrix is also $P$, but the converse in general is not true) [18]. Let us introduce the matrix $\Upsilon$, which is essential in the convergence of distributed algorithms:

$$
[\mathbf{\Upsilon}]_{q r}= \begin{cases}1, & \text { if } q=r \\ -\rho\left(\mathbf{H}_{q q}^{\dagger H} \mathbf{H}_{q r}^{H} \mathbf{H}_{q r} \mathbf{H}_{q q}^{\dagger}\right) \cdot \mathrm{INNR}_{q r}, & \text { if } q \neq r,\end{cases}
$$

where $\mathbf{H}_{q q}$ is assumed to be full column rank, $\mathbf{A}^{\dagger}$ is the MoorePenrose pseudoinverse of $\mathbf{A}, \rho(\mathbf{A})$ is the spectral radius of $\mathbf{A}$, and

$$
\operatorname{INNR}_{q r} \triangleq \frac{\rho\left(\mathbf{R}_{n_{q}}+\sum_{q=1}^{Q} P_{q} \mathbf{H}_{q r} \mathbf{H}_{q r}^{H}\right)}{\lambda_{\min }\left(\mathbf{R}_{n_{q}}\right)} .
$$

To solve the game $\widetilde{\mathcal{G}}_{\mu}$, we propose best-response schemes: all the users, according to a given schedule (e.g., sequentially or simultaneously) solve their own optimization problems (7). The Jacobi version of such a class of algorithms is described in Algorithm 1 below; the more general asynchronous implementation is discussed in [16]. Convergence conditions (valid also for the asynchronous implementation) are given in Theorem 4, whose proof is given in [16].
Theorem 4 The NEP $\widetilde{\mathcal{G}}_{\boldsymbol{\mu}}$ always has a NE. Moreover if $\Upsilon$ is a $P$ matrix, 1) the NE is unique, and 2) the sequence $\left\{\mathbf{Q}^{(n)}, \mathbf{t}^{(n)}\right\}$ generated by Algorithm 1 globally converges to the unique NE.

On the convergence conditions. One sufficient condition for $\Upsilon \Upsilon$ to be $P$ is that there exists a $\mathbf{w}>\mathbf{0}$ such that at least one of the following two conditions is satisfied:

$$
\begin{aligned}
& \frac{1}{w_{q}} \sum_{r \neq q} w_{r}\left\{\rho\left(\mathbf{H}_{q q}^{\dagger H} \mathbf{H}_{q r}^{H} \mathbf{H}_{q r} \mathbf{H}_{q q}^{\dagger}\right) \cdot \operatorname{INNR}_{q r}\right\}<1, \quad \forall q, \\
& \frac{1}{w_{r}} \sum_{q \neq r} w_{q}\left\{\rho\left(\mathbf{H}_{q q}^{\dagger H} \mathbf{H}_{q r}^{H} \mathbf{H}_{q r} \mathbf{H}_{q q}^{\dagger}\right) \cdot \mathrm{INNR}_{q r}\right\}<1, \quad \forall r .
\end{aligned}
$$

The above conditions have an interesting physical interpretation: the uniqueness of the $\mathrm{NE}$ as well as the convergence of best-response algorithms are guaranteed if the interference among the SUs is sufficiently small. Specifically, the first conditions in (12) can be interpreted as a constraint on the maximum amount of interference that each receiver can tolerate, whereas the second conditions impose an upper bound on the maximum amount of interference that each transmitter can generate. Note that the above conditions are independent of the price vector $\boldsymbol{\mu}$.

Implementation issues. Algorithm 1 is a distributed algorithm. Indeed, given the price $\boldsymbol{\mu}$, to compute the best response, each SU only needs to locally measure the covariance matrix of the interference plus noise. To simplify the computational complexity of each bestresponse [the maximum-value function $\phi_{k q}\left(\mathbf{Q}_{q}\right)$ makes the singleuser optimization in (7) intractable], we rewrite each optimization problem in (7) in the following equivalent form, which is amenable for many solvers in the market (as, e.g., SeDuMi [19]):

$$
\begin{array}{cl}
\max _{\mathbf{Q}_{q}, \mathbf{t}_{q}, \boldsymbol{\eta}_{q}} & R_{q}\left(\mathbf{Q}_{q}, \mathbf{Q}_{-q}\right)-\sum_{k=1}^{K} \mu_{k} t_{k q} \\
\text { s.t. } & \mathbf{Q}_{q} \in \mathcal{Q}_{q}, \boldsymbol{\eta}_{q} \geq \mathbf{0}, \\
& {\left[\begin{array}{cc}
\mathbf{A}_{k q}\left(\eta_{k q}, \mathbf{Q}_{q}\right) & -b_{k q}\left(\mathbf{Q}_{q}\right) \\
-b_{k q}\left(\mathbf{Q}_{q}\right)^{H} & c_{k q}\left(t_{k q}, \eta_{k q}, \mathbf{Q}_{q}\right)
\end{array}\right] \succeq \mathbf{0}, \quad \forall k .}
\end{array}
$$

where $\mathbf{A}_{k q}\left(\eta_{k q}, \mathbf{Q}_{q}\right) \triangleq\left(\eta_{k q} \mathbf{T}_{k q}-\mathbf{Q}_{q}\right)^{T} \otimes \mathbf{I}, b_{k q}\left(\mathbf{Q}_{q}\right) \triangleq$ $\operatorname{vec}\left(\widehat{\mathbf{G}}_{k q} \mathbf{Q}_{q}\right), c_{k q}\left(t_{k q}, \eta_{k q}, \mathbf{Q}_{q}\right) \triangleq t_{k q}-\eta_{k q} \varepsilon_{k q}^{2}-\operatorname{tr}\left(\widehat{\mathbf{G}}_{k q} \mathbf{Q}_{q} \widehat{\mathbf{G}}_{k q}^{H}\right)$.

\section{DISTRIBUTED ALGORITHMS FOR $\widetilde{\mathcal{G}}$}

Building on the results in previous sections, we show now how to solve the NCP (11), resulting in distributed algorithms to compute a NE of the original game $\widetilde{\mathcal{G}}$.

Suppose that $\Upsilon$ is a $P$-matrix; then it follows from Theorem 4 and Proposition 3 that the game $\widetilde{\mathcal{G}}$ is equivalent to the NCP (11). We can then focus on algorithms solving the NCP (11). To study convergence of such algorithms, we need the following intermediate property of the mapping $\mathbf{M}(\boldsymbol{\mu})$, proved in [16].

Lemma 5 If $\mathbf{\Upsilon} \succ \mathbf{0}$, there exists a constant $c_{c o c}>0$ such that

$$
\left(\mathbf{M}\left(\boldsymbol{\mu}^{1}\right)-\mathbf{M}\left(\boldsymbol{\mu}^{2}\right)\right)^{T}\left(\boldsymbol{\mu}^{1}-\boldsymbol{\mu}^{2}\right) \geq c_{c o c}\left\|\mathbf{M}\left(\boldsymbol{\mu}^{1}\right)-\mathbf{M}\left(\boldsymbol{\mu}^{2}\right)\right\|_{2}^{2}
$$

for any $\boldsymbol{\mu}^{1} \geq \mathbf{0}$ and $\boldsymbol{\mu}^{2} \geq \mathbf{0}$.

An explicit characterization of $c_{\mathrm{coc}}$ can be found in [16]. The cocoercivity property above is a sufficient condition to guarantee convergence of projection algorithms to solve the NCP (11) [17]. An instance of such algorithms is given in Algorithm 2, and its convergence conditions are in Theorem 6. 
Algorithm 2: Projection Algorithm with Variable Stepsizes

Data: $\boldsymbol{\mu}^{(0)} \geq \mathbf{0}$.

S.0: Set $n=0$.

S.1: If $\boldsymbol{\mu}^{(n)}$ satisfies a termination criterion: STOP.

S.2: Given $\boldsymbol{\mu}^{(n)}$, compute the unique NE of $\widetilde{\mathcal{G}}_{\boldsymbol{\mu}^{(n)}}$ by Algorithm 1.

S.3: Choose a stepsize $\gamma^{(n)}>0$ and update the price vector as

$$
\boldsymbol{\mu}^{(n+1)}=\left[\boldsymbol{\mu}^{(n)}-\gamma^{(n)} \mathbf{M}\left(\boldsymbol{\mu}^{(n)}\right)\right]^{+} .
$$

S.4: $n \leftarrow n+1$ and go back to S.1.

Theorem 6 Suppose that $\mathbf{\Upsilon} \succ \mathbf{0}$ and $0<\gamma^{(n)}<2 c_{c o c}$. Then the sequence $\left\{\boldsymbol{\mu}^{(n)}\right\}$ generated by Algorithm 2 converges to a solution of the NCP (11).

Practical implementation issues. Note that $\mathbf{M}(\boldsymbol{\mu})$ represents the worst-case interference violation at the PUs corresponding to the price value $\boldsymbol{\mu}$. If this quantity was available at the primary receiver, the PUs could then compute the price update (13) and broadcast the new price to the SUs. In practice, however, the PUs can only measure the instantaneous interference, which is typically different from the worst-case interference. The resulting price update becomes

$$
\boldsymbol{\mu}^{(n+1)}=\left[\boldsymbol{\mu}^{(n)}-\gamma^{(n)}\left(\mathbf{M}\left(\boldsymbol{\mu}^{(n)}\right)+\mathbf{e}^{(n)}\right)\right]^{+},
$$

where $\mathbf{e}^{n}$ is an error vector quantifying the deviation of the instantaneous interference violation from the worst-case one.

If the uncertainty region $\mathcal{U}_{k q}$ [cf. (3)] is large enough to include all possible realizations of the real channel $\mathbf{G}_{k q}$, the actual interference is smaller than the worst-case interference and $\mathbf{e}^{(n)} \leq \mathbf{0}$. In this case, there is no guarantee on the convergence of Algorithm 2. We investigate Algorithm 2 with (14) and a constant stepsize $\gamma^{(n)}=$ $\gamma<2 c_{\text {coc }}$ assuming that $\left\|\mathbf{e}^{(n)}\right\| \leq \delta$ for a positive scalar $\delta$.

Proposition 7 Suppose that the error vector $\mathbf{e}^{(n)}$ is bounded such that $\left\|\mathbf{e}^{(n)}\right\|_{2} \leq \delta<+\infty$, and conditions in Theorem 6 are satisfied. Then the sequence $\left\{\boldsymbol{\mu}^{n}\right\}$ generated by Algorithm 2 with (14) under a constant stepsize $\gamma\left(<2 c_{c o c}\right)$ will stay in a bounded neighborhood of $\boldsymbol{\mu}^{\star}$ [the solution of the NCP (11)] specified by

$\left\{\boldsymbol{\mu}:\left\|\boldsymbol{\mu}-\boldsymbol{\mu}^{\star}\right\|_{2}-\sqrt{\left\|\boldsymbol{\mu}-\boldsymbol{\mu}^{\star}\right\|_{2}^{2}-\bar{\gamma}\left\|\mathbf{M}(\boldsymbol{\mu})-\mathbf{M}\left(\boldsymbol{\mu}^{\star}\right)\right\|_{2}^{2}} \leq \gamma \delta\right\}$ where $\bar{\gamma} \triangleq 2 c_{\text {coc }} \gamma-\gamma^{2}$.

If the uncertainty region $\mathcal{U}_{k q}$ is not large enough, the actual interference can sometimes be higher than the theoretic worst-case interference and $\mathbf{e}^{(n)} \geq \mathbf{0}$. Surprisingly, in this case, the effect of error can eventually be eliminated by adopting a proper diminishing stepsize rule for $\gamma^{(n)}$; see [16] for more details.

\section{NUMERICAL RESULTS}

We consider a cellular system composed of one base station, the PU, and 4 secondary communication links. The PU has 4 receive antennas, while the SUs have 4 transmit antennas and 4 receive antennas. We assume equal power budget and white Gaussian noise for all the SUs; the SNR for each SU is $10 \mathrm{~dB}$.

We first compare the robust global approach [Algorithm 2] with the classical nonrobust iterative waterfilling algorithm (IWFA) [Algorithm 1 with $\mu_{k}=0$ in (5) and $\varepsilon_{k q}=0$ in (3)], cf. [20], and the nonrobust global approach [Algorithm 2 with $\varepsilon_{k q}=0$ in (3)]. In

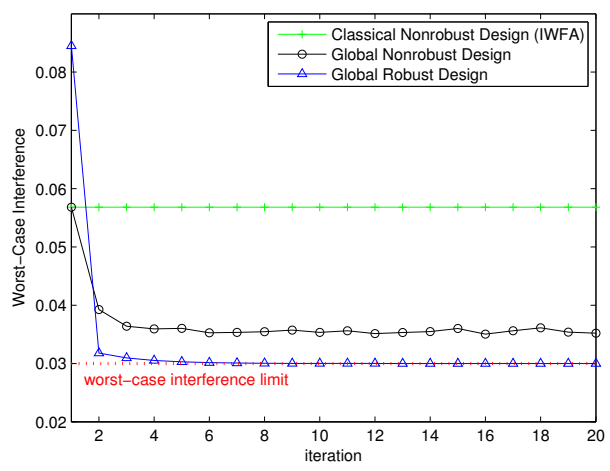

Fig. 1. Worst-case interference versus iteration

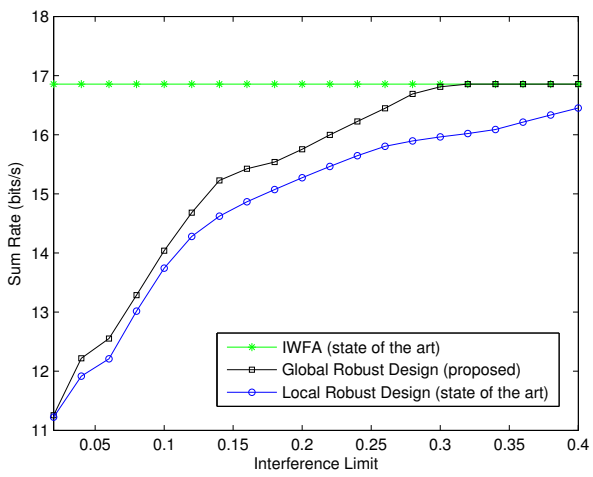

Fig. 2. Sum-rate versus interference limit.

Figure 1 we plot the worst-case interference versus iteration index of the outer loop of Algorithm 2 [with IWFA being the benchmark]. Figure 1 shows that the nonrobust design inevitably result in a violation of the global temperature interference constraint, whereas, the robust global design guarantees that the global temperature interference constraint is always satisfied.

In Figure 2 we compare the performance of the proposed robust global approach [Algorithm 2 with (13)] with the classical IWFA [20] and the robust local approach [9] in terms of sum rate versus interference limit. It is easy to see that the proposed robust global approach outperforms the robust local approach. This is because global interference constraints provide more flexibility than local interference constraints, which instead impose constraints at the level of each SU (the more SUs there are, the more restrictive the local interference constraints would be). In practice, the robust local approach may result in an even worse performance since when the number of SUs is unknown, a conservative estimate would be used, which may be much larger than the real number of SUs there are.

\section{CONCLUSIONS}

In this paper, we have proposed a robust and decentralized design for CR systems composed of multiple primary and secondary users over MIMO IC. The lack of perfect SU-to-PU CSI has been studied under the philosophy of worst-case robustness. We formulated the CR network design as a pricing game, where the SUs play a NEP with given prices and the PUs set prices so that the robust global interference constraints are satisfied at the equilibrium of the game. Building on the theory of complex VIs, we showed that the pricing game always has an equilibrium, and proposed distributed algorithms converging (under technical conditions) to the unique NE of the game. 


\section{REFERENCES}

[1] J. Mitola and G.Q. Maguire, "Cognitive radio: making software radios more personal," IEEE Personal Communications, vol. 6, no. 4, pp. 13-18, 1999.

[2] S. Haykin, "Cognitive radio: brain-empowered wireless communications," IEEE Journal on Selected Areas in Communications, vol. 23, no. 2, pp. 201-220, Feb. 2005.

[3] A. Goldsmith, S.A. Jafar, I. Maric, and S. Srinivasa, "Breaking Spectrum Gridlock With Cognitive Radios: An Information Theoretic Perspective," Proceedings of the IEEE, vol. 97, no. 5, pp. 894-914, May 2009.

[4] Gesualdo Scutari and Daniel P. Palomar, "MIMO Cognitive Radio: A Game Theoretical Approach," IEEE Transactions on Signal Processing, vol. 58, no. 2, pp. 761-780, Feb. 2010.

[5] Jong-Shi Pang, Gesualdo Scutari, Daniel P. Palomar, and Francisco Facchinei, "Design of Cognitive Radio Systems Under Temperature-Interference Constraints: A Variational Inequality Approach," IEEE Transactions on Signal Processing, vol. 58, no. 6, pp. 3251-3271, June 2010.

[6] G. Scutari, D. P. Palomar, F. Facchinei, and J. Pang, "Monotone games for cognitive radio systems," in Distributed DecisionMaking and Control, Anders Rantzer and Rolf Johansson, Eds. Springer Verlag, 2011.

[7] Lan Zhang, Ying-Chang Liang, and Yan Xin, "Joint Beamforming and Power Allocation for Multiple Access Channels in Cognitive Radio Networks," IEEE Journal on Selected Areas in Communications, vol. 26, no. 1, pp. 38-51, Jan. 2008.

[8] Seung-Jun Kim and Georgios B. Giannakis, "Optimal Resource Allocation for MIMO Ad Hoc Cognitive Radio Networks," IEEE Transactions on Information Theory, vol. 57, no. 5, pp. 3117-3131, May 2011.

[9] Jiaheng Wang, Gesualdo Scutari, and Daniel P. Palomar, "Robust MIMO Cognitive Radio Via Game Theory," IEEE Transactions on Signal Processing, vol. 59, no. 3, pp. 1183-1201, Mar. 2011.

[10] K.T. Phan, S.A. Vorobyov, N.D. Sidiropoulos, and C. Tellambura, "Spectrum sharing in wireless networks via qos-aware secondary multicast beamforming," IEEE Transactions on Signal Processing, vol. 57, no. 6, pp. 2323-2335, June.

[11] Gesualdo Scutari, Francisco Facchinei, Jong-Shi Pang, and Daniel P. Palomar, "Real and Complex Monotone Communication Games," Submitted to IEEE Transactions on Information Theory, Available: http://arxiv.org/abs/1212.6235, 2012.

[12] Y.C. Eldar and N. Merhav, "A Competitive Minimax Approach to Robust Estimation of Random Parameters," IEEE Transactions on Signal Processing, vol. 52, no. 7, pp. 1931-1946, July 2004.

[13] Jiaheng Wang and Daniel P. Palomar, "Worst-Case Robust MIMO Transmission With Imperfect Channel Knowledge," IEEE Transactions on Signal Processing, vol. 57, no. 8, pp. 30863100, Aug. 2009.

[14] Lan Zhang, Ying-Chang Liang, Yan Xin, and H.V. Poor, "Robust cognitive beamforming with partial channel state information," IEEE Transactions on Wireless Communications, vol. 8, no. 8, pp. 4143-4153, Aug. 2009.
[15] Gan Zheng, Kai-Kit Wong, and B. Ottersten, "Robust Cognitive Beamforming With Bounded Channel Uncertainties," IEEE Transactions on Signal Processing, vol. 57, no. 12, pp. 4871-4881, Dec. 2009.

[16] Yang Yang, Peiran Song, Gesualdo Scutari, and Daniel P. Palomar, "Robust MIMO Cognitive Radio Systems Under Temperature Interference Constraints," Submitted to IEEE Journal on Selected Areas in Communications, 2012.

[17] Francisco Facchinei and Jong-Shi Pang, Finite-dimensional variational inequalities and complementarity problems, Springer-Verlag, New York; Hong Kong, 2003.

[18] Richard Cottle, Jong-Shi Pang, and Richard E. Stone, The linear complementarity problem, Academic Press, Boston, 1992, Richard W. Cottle, Jong-Shi Pang, Richard E. Stone; :ill. ;24 $\mathrm{cm}$; Includes bibliographical references and index.

[19] Jos F. Sturm, "Using SeDuMi 1.02, A Matlab toolbox for optimization over symmetric cones," Optimization Methods and Software, vol. 11, no. 1-4, pp. 625-653, Jan. 1999.

[20] Gesualdo Scutari, Daniel P. Palomar, and Sergio Barbarossa, "The MIMO Iterative Waterfilling Algorithm," IEEE Transactions on Signal Processing, vol. 57, no. 5, pp. 1917-1935, May 2009. 